\title{
Classical Properties on Conformable Fractional Calculus
}

\author{
Musraini M.*, Rustam Efendi, Endang Lily, Ponco Hidayah
}

Department of Mathematics, University of Riau, Pekanbaru, Indonesia

\section{Email address:}

musraini.m4r@gmail.com (Musraini M.),rustam.r4m@gmail.com (Rustam E.),endanglily60@gmail.com (Endang L.), ponhid21@gmail.com (Ponco H.)

${ }^{*}$ Corresponding author

\section{To cite this article:}

Musraini M., Rustam Efendi, Endang Lily, Ponco Hidayah. Classical Properties on Conformable Fractional Calculus. Pure and Applied Mathematics Journal. Vol. 8, No. 5, 2019, pp. 83-87. doi: 10.11648/j.pamj.20190805.11

Received: September 3, 2019; Accepted: October 9, 2019; Published: October 23, 2019

\begin{abstract}
Recently, a definition of fractional which refers to classical calculus form called conformable fractional calculus has been introduced. The main idea of the concept of conformable fractional calculus is how to determine the derivative and integral with fractional order either rational numbers or real numbers. One of the most popular definitions of conformable fractional calculus is defined by Katugampola which is used in this study. This definition satisfies in some respects of classical calculus involved conformable fractional derivative and conformable fractional integral. In the branch of conformable fractional derivatives, some of the additional results such as analysis of fractional derivative in quotient property, product property and Rolle theorem are given. An application on classical calculus such as determining monotonicity of function is also given. Then, in the case of fractional integral, this definition showed that the fractional derivative and the fractional integral are inverses of each other. Some of the classical integral properties are also satisfied on conformable fractional integral. Additionally, this study also has shown that fractional integral acts as a limit of a sum. After that, comparison properties on fractional integral are provided. Finally, the mean value theorem and the second mean value theorem are also applicable for fractional integral.
\end{abstract}

Keywords: Fractional Calculus, Conformable Fractional Derivative, Conformable Fractional Integral

\section{Introduction}

Today various types of fractional calculus have been proposed by many researchers. The most popular definition is given by the Riemann-Liouville, Caputo, Grünwald Letnikov, Hadamard definition. The information about their definition can be found in $[12,14,15]$. Most of the types of fractional calculus definitions that have been introduced cannot be used for classical properties such as product rules, quotient rules, chain rules, Rolle theorems, and mean value theorems. Therefore, Khalil et al. [11] introduced a new modest idea. The definition called conformable fractional calculus is the definition of fractional derivative and integral with $\alpha \in(0,1)$ order and it satisfies classical properties mentioned above. Moreover, there are several researchers introduced their definition conformable fractional calculus in the other form [1-3, 10]. Recently, the concept of conformable fractional calculus has gained relevance, namely because they kept some of the properties of ordinary derivatives. Even more, this new subject has been important topics to discuss because there are several applications about this topic [5, 16]. Also, further results about this subject were developed by [6]. The aim of this paper is to provide additional results of conformable fractional calculus based on the definition introduced by Katugampola [10]. There are several properties of conformable fractional calculus that well functioned, like classical calculus involving fractional derivative and fractional integral, such as properties to determine fractional derivatives, definite fractional integral as the limit of a sum and comparison properties of fractional integrals are also developed. Aditionally, some applications are given.

\section{Conformable Fractional Derivative}

Katugampola [10] introduced natural definition of fractional derivative definition which satisfies classical derivative properties. 


\subsection{Definition of Conformable Fractional Derivative}

Let $a \geq 0$ and $\alpha \in(0,1)$. Given a function $f:[a, b] \rightarrow \mathbb{R}$. Then conformable fractional derivative of $f$ is defined by

$$
D^{\alpha}(f)(x)=\lim _{\epsilon \rightarrow 0} \frac{f\left(x e^{\epsilon x^{-\alpha}}\right)-f(x)}{\epsilon},
$$

for $x>0$. If $f$ be $\alpha$-differentiable on $(0, a), a>0$ and $\lim _{X \rightarrow 0^{+}} f^{\alpha}(x)$ exists. Then, $f^{\alpha}(0)=\lim _{x \rightarrow 0^{+}} f(x)$.

The following theorem is an important result to prove the next consequences.

\subsection{The Result Theorem of Conformable Fractional Derivative}

If $b>a>0$ and $f:[a, b] \rightarrow \mathbb{R}$ is differentiable function then $f$ is $\alpha$-differentiable function at $x>a$, then

$$
f^{\alpha}(x)=x^{1-\alpha} \frac{d}{d x} f(x) .
$$

Proof. Using (1) and taking $h=\epsilon x^{1-\alpha}(1+O(\epsilon)$ then,

$$
\begin{gathered}
D^{\alpha}(f)(x)=\lim _{\epsilon \rightarrow 0} \frac{f\left(x e^{\epsilon x^{-\alpha}}\right)-f(x)}{\epsilon}, \\
=\lim _{\epsilon \rightarrow 0} \frac{f\left(x+\epsilon x^{1-\alpha}+O(\epsilon)\right)-f(x)}{\epsilon} \\
=\lim _{\epsilon \rightarrow 0} \frac{f(x+h)-f(x)}{\frac{h x^{\alpha-1}}{1+O(\epsilon)}} \\
D^{\alpha}(f)(x)=x^{1-\alpha} \frac{d}{d x} f(x),
\end{gathered}
$$

since by assumption, $f$ is differentiable at $x>0$. This completes the proof of the theorem.

\subsection{Properties of Conformable Fractional Derivative}

Let $\alpha \in(0,1]$ and $f, g$ be $\alpha$-differentiable at a point $x>0$. Then
i. $D^{\alpha}(c)=0 . c \in \mathbb{R}$
ii. $D^{\alpha}(a f+b g)(x)=a D^{\alpha}(f)(x)+b D^{\alpha}(g)(x)$.
iii. $D^{\alpha}(f g)(x)=g(x) D^{\alpha}(f)(x)+f(x) D^{\alpha}(g)(x)$.
iv. $D^{\alpha}\left(\frac{f}{g}\right)(x)=\frac{g(x) D^{\alpha}(f)(x)-f(x) D^{\alpha}(g)(x)}{[g(x)]^{2}}$.
v. $D^{\alpha}(f \circ g)(x)=f^{\prime}(g(x)) D^{\alpha}(g)(x)$ (Chain Rule).

Proof. Using (2), all properties will be proven consecutively. Now, for fixed $\alpha \in(0,1]$, it is easily seen that $x^{1-\alpha} \frac{d}{d x}(c)=$ $x^{1-\alpha} \cdot 0=0$. This is prove of property (i). Secondly, for the property (ii),

$$
\begin{gathered}
D^{\alpha}(a f+b g)(x)=x^{1-\alpha}(a f+b g)^{\prime}(x) \\
=x^{1-\alpha}(a f+b g)^{\prime}(x) \\
=x^{1-\alpha}\left(a f^{\prime}(x)+b g^{\prime}(x)\right) \\
=x^{1-\alpha} a f^{\prime}(x)+x^{1-\alpha} b g^{\prime}(x) .
\end{gathered}
$$

Hence, the definition satisfies linearity property. Using similar arguments applied on property (ii), then property (iii) is proven by

$$
\begin{gathered}
D^{\alpha}(f g)(x)=x^{1-\alpha}(f g)^{\prime}(x) \\
=x^{1-\alpha}\left(f^{\prime} g+f g^{\prime}\right)(x) \\
=x^{1-\alpha}\left(f^{\prime} g\right)(x)+x^{1-\alpha}\left(f g^{\prime}\right)(x) \\
=x^{1-\alpha} f^{\prime}(x) g(x)+x^{1-\alpha} f(x) g^{\prime}(x) \\
D^{\alpha}(f g)(x)=\left(D^{\alpha} f(x)\right) g(x)+\left(D^{\alpha} g(x)\right) f(x)
\end{gathered}
$$

Then, for (iv)

$$
\begin{gathered}
D^{\alpha}\left(\frac{f}{g}\right)(x)=x^{1-\alpha}\left(\frac{f}{g}\right)^{\prime}(x) \\
D^{\alpha}\left(\frac{f}{g}\right)(x)=x^{1-\alpha} \frac{\left(g(x) f^{\prime}(x)-f(x) g^{\prime}(x)\right)}{[g(x)]^{2}} \\
=\frac{\left(g(x) f^{\prime}(x) x^{1-\alpha}-f(x) g^{\prime}(x) x^{1-\alpha}\right)}{[g(x)]^{2}} \\
D^{\alpha}\left(\frac{f}{g}\right)(x)=\frac{g(x) D^{\alpha}(f)(x)-f(x) D^{\alpha}(g)(x)}{[g(x)]^{2}}
\end{gathered}
$$

Finally, property (v) will be proven using (2) as the following

$$
\begin{gathered}
D^{\alpha}(f \circ g)(x)=x^{1-\alpha}(f \circ g)^{\prime}(x) \\
\quad=x^{1-\alpha} f^{\prime}\left(g(x) g^{\prime}(x)\right. \\
\quad=f^{\prime}(g(x)) x^{1-\alpha} g^{\prime}(x) \\
D^{\alpha}(f \circ g)(x)=f^{\prime}(g(x))\left(D^{\alpha}(g(x))\right.
\end{gathered}
$$

This completed the proof of the theorem.

\subsection{Corollary of Quotient Property}

Let $\alpha \in(0,1]$ and $f, g$ be $\alpha$-differentiable at a point $x>0$. Then

$$
D^{\alpha}\left(\frac{1}{f(x)}\right)=-\frac{f^{\alpha}(x)}{[f(x)]^{2}} .
$$

Proof. Using (2) and the property (iv) of theorem 2.3, then

$$
\begin{gathered}
D^{\alpha}\left(\frac{1}{f(x)}\right)=x^{1-\alpha}\left(\frac{1}{f(x)}\right)^{\prime} \\
=x^{1-\alpha} \frac{\left(f(x)(0)-f^{\prime}(x)\right)}{[f(x)]^{2}} \\
=-\frac{f^{\prime}(x) x^{1-\alpha}}{[f(x)]^{2}} \\
D^{\alpha}\left(\frac{1}{f(x)}\right)=-\frac{f^{\alpha}(x)}{[f(x)]^{2}} .
\end{gathered}
$$

\subsection{Corollary of Product Property}

Let $\alpha \in(0,1]$ and $f, g$ be $\alpha$-differentiable at a point 
$x>0$. Then

$$
D^{\alpha}(f(x))^{2}=2\left(f(x) f^{\alpha}(x)\right)
$$

Proof. Using (2) and the property (iii) of theorem 2.3, then

$$
\begin{gathered}
D^{\alpha}(f(x))^{2}=x^{1-\alpha}\left(f^{\prime(x)}\right)^{2} \\
=x^{1-\alpha}\left(f(x) f^{\prime}(x)+f^{\prime}(x) f(x)\right) \\
=\left(x^{1-\alpha} f(x) f^{\prime}(x)\right)+\left(x^{1-\alpha} f^{\prime}(x) f(x)\right) \\
D^{\alpha}(f(x))^{2}=f(x) f^{\alpha}(x)+f^{\alpha}(x) f(x) \\
D^{\alpha}(f(x))^{2}=2\left(f(x) f^{\alpha}(x)\right)
\end{gathered}
$$

\subsection{Rolle Theorem for Fractional Derivative}

Let $a>0$ and $f:[a, b] \rightarrow \mathbb{R}$ be a function satisfying the following

i. $f$ continuous on $[a, b]$,

ii. $f$ is $\alpha$-differentiable for some $\alpha \in(0,1)$,

iii. $f(a)=f(b)$,

Then, there extists $c \in(a, b)$ such that $f^{(\alpha)}(c)=0$.

Proof. Since $f$ is continuous on $[a, b]$ and $f(a)=f(b)$, there exists $c \in(a, b)$ at which the function has a local extrema. Then

$$
\begin{aligned}
& D^{\alpha}(f(c))=\lim _{\epsilon \rightarrow 0^{-}} \frac{f\left(c e^{\epsilon c^{-\alpha}}\right)-f(c)}{\epsilon} \\
& D^{\alpha}(f(c))=\lim _{\epsilon \rightarrow 0^{+}} \frac{f\left(c e^{\epsilon c^{-\alpha}}\right)-f(c)}{\epsilon}
\end{aligned}
$$

But, the two limits have opposite sign, so $D^{\alpha}(f(c))=0$. Consider the function

$$
F(x)=f(x)-f(a)-\frac{f(b)-f(a)}{\frac{1}{\alpha} b^{\alpha}-\frac{1}{\alpha} a^{\alpha}}\left(\frac{1}{\alpha} x^{\alpha}-\frac{1}{\alpha} a^{\alpha}\right) .
$$

Then, the function $g$ satisfies the conditions of the fractional Rolle's theorem. Hence there exists $c \in(a, b)$, such that $D^{\alpha}\left(\frac{1}{\alpha} x^{\alpha}\right)=1$, the result follows.

\subsection{Monotonicity}

Let $a>0$ and $f:[a, b] \rightarrow \mathbb{R}$ be $\alpha$-differentiable on an interval $[a, b]$.

i. If $f^{\alpha}(x) \geq 0$ for all $x \in[a, b]$, then $f$ is nondecreasing on $[a, b]$.

ii. If $f^{\alpha}(x)>0$ for all $x \in[a, b]$, then $f$ is increasing on $[a, b]$.

iii. If $f^{\alpha}(x) \leq 0$ for all $x \in[a, b]$, then $f$ is nonincreasing on $[a, b]$.

iv. If $f^{\alpha}(x)<0$ for all $x \in[a, b]$, then $f$ is decreasing on $[a, b]$.

v. If $f^{\alpha}(x)=0$ for all $x \in[a, b]$, then $f$ is constant on $[a, b]$.

Proof. For part (i), let $x_{1}, x_{2} \in[a, b]$ with $x_{1}<x_{2}$. Using theorem 2.7 there exists $c \in\left(x_{1}, x_{2}\right)$ such that

$$
f\left(x_{2}\right)-f\left(x_{1}\right)=f^{\alpha}(c)\left(\frac{1}{\alpha} x^{\alpha}-\frac{1}{\alpha} a^{\alpha}\right) .
$$

If $f^{\alpha}(c) \geq 0$ then $f\left(x_{2}\right) \geq f\left(x_{1}\right)$. Therefore, if $f^{\alpha}(x) \geq 0$ for all $x \in[a, b]$, then $f$ is nondecreasing on $[a, b]$. For parts (ii), (iii), (iv) can be proved with similar arguments on part (i). Then part (v) follows immediately from (i) and (iii) such that $f^{\alpha}(x)=0$ be a constant, neither decreasing or increasing.

\section{Conformable Fractional Integral}

The conformable fractional integral is discussed as follows.

\subsection{Definition of Conformable Fractional Integral}

Let $a \geq 0$, and $\alpha \in(0,1)$. Also, let $f$ be a continuous function such that $I^{\alpha} f$ exists. Then

$$
I_{\alpha}^{a}(f(x))=\int_{a}^{x} \frac{f(t)}{t^{1-\alpha}} d t
$$

If the Riemann improper integral exists.

This following theorem explains that $\alpha$ - fractional derivative and $\alpha$-fractional integral are inverse of each other as given in the next result.

\subsection{Inverse Property}

Let $a \geq 0$ and $\alpha \in(0,1)$. Also, let $f$ be a continuous function such that $I^{\alpha}(f(x))$ exists. For all $x>a$, then

$$
D^{\alpha}\left[I^{\alpha} f(x)\right]=f(x) .
$$

Proof. Since $f$ is continuous, then $I^{\alpha}(f)$ is certainly differentiable. Using theorem 2.2, then

$$
\begin{gathered}
D^{\alpha}\left[I^{\alpha}(f(x))\right]=x^{1-\alpha} \frac{d}{d x}\left[I^{\alpha}(f(x))\right], \\
=x^{1-\alpha} \frac{d}{d x} \int_{a}^{x} \frac{f(t)}{t^{1-\alpha}} d t \\
=x^{1-\alpha} \frac{f(x)}{x^{1-\alpha}} \\
D^{\alpha}\left[I^{\alpha} f(x)\right]=f(x)
\end{gathered}
$$

\subsection{Conformable Fractional Integral of Conformable Fractional Derivative}

Let $f:(a, b) \rightarrow R$ be $\alpha$-differentiable and $0<\alpha \leq 1$. For all $x>a$ then

$$
I^{\alpha}\left[D^{\alpha}(f)(x)\right]=f(x)-f(a) .
$$

Proof. From definition 3.1, it is easily seen that

$$
\begin{gathered}
I^{\alpha}\left[D^{\alpha}(f)(x)\right]=\int_{a}^{x} D^{\alpha}(f(t)) d t \\
=\int_{a}^{x} t^{1-\alpha} t^{\alpha-1}(f(t)) d t
\end{gathered}
$$




$$
\begin{gathered}
=\int_{a}^{x} f(t) d t \\
I^{\alpha}\left[D^{\alpha}(f)(x)\right]=f(x)-f(a)
\end{gathered}
$$

The following a definition of integration as a limit of a sum is provided. This definition has a number of benefits which are reviewed below.

\subsection{Conformable Fractional Integral as a Limit of a Sum}

If $f$ is a function defined for $a<x \leq b$, Then the definite fractional integral of $f$ from $a$ to be $b$ is

$$
\int_{a}^{b} \frac{f(x)}{x^{1-\alpha}} d x=\lim _{n \rightarrow \infty} \sum_{i=1}^{n} \frac{f\left(x_{i}\right)}{x_{i}^{1-\alpha}} \Delta x .
$$

Where $\Delta x=(b-a) / n$ and $x_{i}=a+i \Delta x$.

\subsection{Conformable Fractional Integral Properties}

If $a \geq 0, \alpha \in(0,1)$ and $f, g:[a, b] \rightarrow \mathbb{R}$ be a continuous function. Then
i. $\int_{a}^{b} \frac{\lambda}{x^{1-\alpha}} d x=$$$
\lambda\left(\left[\frac{\left(\alpha-1 \cdot b^{(\alpha-1)+1}\right)}{\alpha-1+1}\right]-\left[\frac{\left(\alpha-1 \cdot a^{(\alpha-1)+1}\right)}{\alpha-1+1}\right]\right), \lambda \in \mathbb{R}
$$$$
\text { ii. } \int_{a}^{b} \frac{(f(x)+g(x))}{x^{1-\alpha}} d x=\int_{a}^{b} \frac{f(x)}{x^{1-\alpha}} d x+\int_{a}^{b} \frac{g(x)}{x^{1-\alpha}} d x
$$$$
\text { iii. } \int_{a}^{b} \lambda \frac{f(x)}{x^{1-\alpha}} d x=\lambda \int_{a}^{b} \frac{f(x)}{x^{1-\alpha}} d x, \lambda \in \mathbb{R} \text {, }
$$$$
\text { iv. } \int_{a}^{b} \frac{f(x)}{x^{1-\alpha}} d x=-\int_{a}^{b} \frac{f(x)}{x^{1-\alpha}} d x
$$$$
\text { v. } \int_{a}^{b} \frac{f(x)}{x^{1-\alpha}} d x=\int_{a}^{c} \frac{f(x)}{x^{1-\alpha}} d x+\int_{c}^{b} \frac{f(x)}{x^{1-\alpha}} d x
$$$$
\text { vi. } \int_{a}^{a} \frac{f(x)}{x^{1-\alpha}} d x=0 \text {. }
$$

Proof. It is easy to proof property (i). Since it is known that integral of constant functions is multiplication $c$ with integral integral of power function. Property (ii) is proved using definition 3.4 as the following.

$$
\begin{gathered}
\int_{a}^{b} \frac{f(x)+g(x)}{x^{1-\alpha}} d x=\lim _{n \rightarrow \infty} \sum_{i=1}^{n}\left[\frac{f\left(x_{i}\right)+g(x)}{x_{i}{ }^{1-\alpha}}\right] \Delta x, \\
=\lim _{n \rightarrow \infty} \sum_{i=1}^{n}\left[\frac{f\left(x_{i}\right)}{x_{i}{ }^{1-\alpha}}\right] \Delta x+\lim _{n \rightarrow \infty} \sum_{i=1}^{n}\left[\frac{g(x)}{x_{i}{ }^{1-\alpha}}\right] \Delta x, \\
\int_{a}^{b} \frac{f(x)+g(x)}{x^{1-\alpha}} d x=\int_{a}^{b} \frac{f(x)}{x^{1-\alpha}} d x+\int_{a}^{b} \frac{g(x)}{x^{1-\alpha}} d x
\end{gathered}
$$

Part (iii), (iv), (v), (vi) can be proven similarly with arguments on part (ii).

\subsection{Comparison Properties of Conformable Fractional Integral}

Let $a \geq 0$ and $\alpha \in(0,1]$. Also, let $f, g:[a, b] \rightarrow \mathbb{R}$ be a continuous function. Then,

i. If $f(x) \geq 0$ for all $x \in[a, b]$, then $\int_{a}^{b} \frac{f(x)}{x^{1-\alpha}} d x \geq 0$.

ii. If $f(x) \geq g(x)$ for all $x \in[a, b]$, then $\int_{a}^{b} \frac{f(x)}{x^{1-\alpha}} d x \geq$ $\int_{a}^{b} \frac{g(x)}{x^{1-\alpha}} d x$. iii. $\left|\int_{a}^{b} \frac{f(x)}{x^{1-\alpha}} d x\right| \leq \int_{a}^{b} \frac{|f(x)|}{x^{1-\alpha}} d x$.

If $f$ is continuous and $m \leq f(x) \leq M$ for all $x \in[a, b]$, then

$$
m \int_{a}^{b} \frac{g(x)}{x^{1-\alpha}} d x \leq \int_{a}^{b} \frac{f(x) g(x)}{x^{1-\alpha}} d x \leq M \int_{a}^{b} \frac{g(x)}{x^{1-\alpha}} d x,
$$

for any continuous nonnegative $g$.

Proof. Firstly, property (i) is proven by definition 3.4 such that

$$
\int_{a}^{b} \frac{f(x)}{x^{1-\alpha}} d x=\lim _{n \rightarrow \infty} \sum_{i=1}^{n} \frac{f\left(x_{i}\right)}{x_{i}{ }^{1-\alpha}} \Delta x
$$

Now, by assumption $f(x) \geq 0$ and $\Delta x \geq 0$. It is known that

$$
\lim _{n \rightarrow \infty} \sum_{i=1}^{n} \frac{f\left(x_{i}\right)}{x_{i}^{1-\alpha}} \Delta x \geq 0
$$

Based on the basic properties of limits, it can be concluded that

$$
\lim _{n \rightarrow \infty} \sum_{i=1}^{n} \frac{f\left(x_{i}\right)}{x_{i}^{1-\alpha}} \Delta x \geq \lim _{n \rightarrow \infty} 0=0 .
$$

As it is known, the left side is surely the definition of the conformable fractional integral. Therefore the following is true.

$$
\int_{a}^{b} \frac{f(x)}{x^{1-\alpha}} d x \geq 0
$$

For property (ii), since $f(x) \geq g(x)$, then that $f(x)-$ $g(x) \geq 0$ on $a<x \leq b$. So, using property (i) above and property (ii) on theorem 3.5 it is easily seen that,

$$
\int_{a}^{b} \frac{f(x)}{x^{1-\alpha}} d x-\int_{a}^{b} \frac{g(x)}{x^{1-\alpha}} d x \geq 0 .
$$

Thus,

$$
\int_{a}^{b} \frac{f(x)}{x^{1-\alpha}} d x \geq \int_{a}^{b} \frac{g(x)}{x^{1-\alpha}} d x
$$

The last property is (iii). It is equivalent with

$$
-\int_{a}^{b} \frac{|f(x)|}{x^{1-\alpha}} d x \leq \int_{a}^{b} \frac{f(x)}{x^{1-\alpha}} d x \leq \int_{a}^{b} \frac{|f(x)|}{x^{1-\alpha}} d x .
$$

This follows immediately from property (i) and (ii) because

$$
-|f(x)| \leq f(x) \leq f(x) .
$$

Finally, property (iv) can be proven by using property (ii) on theorem 3.6 .

\subsection{Mean Value Theorem for Fractional Integral}

If $f:[a, b] \rightarrow \mathbb{R}$ is a continuous function on $[a, b]$. Then, there exists $c$ in $[a, b]$ such that, 


$$
f(c)=\left(\frac{1}{\alpha} b^{\alpha}-\frac{1}{\alpha} a^{\alpha}\right) \int_{a}^{b} \frac{f(x)}{x^{1-\alpha}} d x .
$$

Proof. Define

$$
I^{\alpha} f(x)=\int_{a}^{x} \frac{f(t)}{t^{1-\alpha}} d t
$$

Since $f(x)$ is continuous and recall that from theorem 3.2 $I^{\alpha} f(x)$ is continuous on $[a, b], \alpha$-differentiable on $(a, b)$ and $D^{\alpha}\left[I^{\alpha} f(x)\right]=f(x)$. Now, from the theorem 2.7 it can be stated that there is a number $c$ such that $a<c<b$ and

$$
I^{\alpha} f(b)-I^{\alpha} f(a)=D^{\alpha}\left[I^{\alpha} f(x)\right]\left(\frac{1}{\alpha} b^{\alpha}-\frac{1}{\alpha} a^{\alpha}\right) .
$$

However, it is known that $D^{\alpha}\left[I^{\alpha} f(c)\right]=f(c)$ and

$I^{\alpha} f(b)=\int_{a}^{b} \frac{f(t)}{t^{1-\alpha}} d t=\int_{a}^{b} \frac{f(x)}{x^{1-\alpha}} d x, I^{\alpha} f(a)=\int_{a}^{a} \frac{f(x)}{x^{1-\alpha}} d x=0$

Thus

$$
\int_{a}^{b} \frac{f(x)}{x^{1-\alpha}} d x=f(c)\left(\frac{1}{\alpha} b^{\alpha}-\frac{1}{\alpha} a^{\alpha}\right) .
$$

\subsection{Second Mean Value Theorem for Fractional Integral}

Let $f$ and $g$ be functions satisfying the following

Continuous on $[a, b]$,

Bounded and integrable on $[a, b]$,

$m=\inf \{f(x): x \in[a, b]\}$ and $M=\sup \{f(x): x \in[a, b]$.

Then, there exists a number $c \in(a, b)$ such that

$$
\int_{a}^{b} \frac{f(x) g(x)}{x^{1-\alpha}} d x \leq c \int_{a}^{b} \frac{g(x)}{x^{1-\alpha}} d x .
$$
then

Proof. If $m=\inf f, M=\sup f$ and $g(x) \geq 0$ in $[a, b]$,

$$
m g(x)<f(x) g(x)<M g(x)
$$

Multiply (3) by $x^{1-\alpha}$ and integrate (3) with respect to $x$ over $(a, b)$, resulting

$$
m \int_{a}^{b} \frac{g(x)}{x^{1-\alpha}} d x \leq \int_{a}^{b} \frac{f(x) g(x)}{x^{1-\alpha}} d x \leq M \int_{a}^{b} \frac{g(x)}{x^{1-\alpha}} d x .
$$

Then there exists a number $c$ in $[m, M]$ such that

$$
\int_{a}^{b} \frac{f(x) g(x)}{x^{1-\alpha}} d x \leq c \int_{a}^{b} \frac{g(x)}{x^{1-\alpha}} d x .
$$

\section{Conclusion}

In this note, further properties of conformable fractional calculus involving fractional derivative and integral are provided. This definition satisfies several properties and applications of classical calculus such as the rules to determine conformable fractional derivative and fractional integral, mean value theorem and comparison properties of conformable fractional integral. Some applications are also given.

\section{References}

[1] T. Abdeljawad, On conformable fractional calculus, Journal of Computational and Applied Mathematics, (279), 57-66 (2015).

[2] A. Akkurt, M. E. Yildirim dan H. Yildirim, Generalized new fractional derivative and integral, Konuralp Journal of Mathematics, (5), 248-259 (2017).

[3] R. Almeida, M. Guzowska dan T. Odzijewicz, A remark on local fractional calculus and ordinary derivatives, Open Mathematics Research Article, (14), 1122-1124 (2016).

[4] A. Atangana, D. Baleanu, New fractional derivatives with nonlocal and non-singular kernel: Theory and application to heat transfer model, arXiv: 1602.03408 [math. GM] 2016.

[5] R. Anderson and D. J. Ulness, Properties of the Katugampola fractional derivative with potential applicationin quantum mechanics, Journal of Mathematical Physics, 56 (2015).

[6] O. T. Birgani, S. Chandok, N. Dedović dan S. Radenović, A note on some recent results of theconformable derivative, Advances in the Theory of Nonlinear Analysis and its Applications, (3), 11-17 (2019).

[7] C.-Q. Dai, Y. Wang, J. Liu, Spatiotemporal Hermite-Gaussian solitons of a $(3+1)$-dimensional partially nonlocal nonlinear Schrodinger equation. Nonlinear Dynamics, (84), 1157-1161 (2016).

[8] J. Hadamard, Essai sur l'étude des fonctions donnés par leur développement de Taylor, Journalde Mathématiques Pures et Appliquées, (8), 101-186 (1892).

[9] Hermann, Richard, Fractional Calculus: An Introduction for Physicists Analysis Second Edition, New Jersey, World Scientific Publishing, 2014.

[10] U. Katumgapola, A new fractional derivative with classical properties, preprint, arXiv: 1410.6535.

[11] R. Khalil, M. Alhorani, A. Yousef dan M. Sababheh, A definition of fractional derivative, Journal of Computational Applied Mathematics, (264), 65-70 (2014).

[12] B. Ross, The development of fractional calculus 1695-1900, Historia Mathematica, (4), 75-89 (1977).

[13] N. A. Shah and A. F. I. Khan, Heat transfer analysis in a second grade fluid over and oscillating vertical plate using fractional Caputo-Fabrizio derivatives, The European Physical Journal C. 76, (7), 1-11 (2016).

[14] N. A. Sheikh, A. F. I. Khan, M. Saqib, A modern approach of Caputo-Fabrizio time-fractionalderivative to MHD free convection flow of generalized second-grade fluid in a porous medium, Neural Computing and Applications, 1-11 (2016).

[15] M. H. Tavassoli, A. Tavassoli dan M. R. O. Rahimi, The geometric and physical interpretationof fractional order derivative of polynomial functions, Differential Geometry-Dynamical System, (15), 93-104 (2013).

[16] F. Usta, A conformable calculus of radial basis functions and its applications, An International journal of optimization and control: Theories and Applications, (8), 176-182 (2018). 